\title{
The Profound Unravelling of Equilibrium Thermodynamics and Arrhenius First-Order Kinetics in Relation to Metal Hydride Desorption Reactions
}

Roland H. Pawelke

\begin{abstract}
Approaching the entanglement problem of kinetics with thermodynamics in reversible metal hydride desorption reactions by means of a hyperbola template such as the Michaelis-Menten curve renders a closed solution for their unravelling possible, revealing profound insight of general significance into both, the structure of the rate-limiting thermodynamic factor and the nature of experiment-specific first-order Arrhenius kinetics. As by-product an alternate method of extreme simplicity for modelling transient behaviour of reversible metal hydride tanks is obtained. This paper concludes a series of works concerned with objectively approaching metal hydride soprtion reaction kinetics.
\end{abstract}

\section{Introduction}

Chemical hydrogen storage in reversible metal hydrides offers a means for reducing the $p V$-energy inside a pressurized vessel supplying a fuel cell. ${ }^{1,2}$ Since the release of hydrogen from a reversible metal hydride consumes heat, this can be exploited for reducing the waste heat load of a fuel cell energy system. ${ }^{3-5}$ Hydrogen gas at typical metal hydride sorption reaction conditions ( $T>300 \mathrm{~K}, p<100$ bar) may be well approximated as ideal, at $300 \mathrm{~K}$ and 100 bar is the deviation from the van der Waals equation $6.8 \%$ with regard to molar volume. The fundamental nature of reversible chemical hydrogen storage is those of a thermodynamic two-phase gas-sorbent equilibrium system: because it has only one degree of freedom according to the phase rule of Gibbs, either temperature or pressure (in the tank) may be set freely - the other quantity adjusts accordingly. This principle is the lever for mediating process direction and the selfregulating nature has also a profound effect on the kinetics of hydrogen release: ${ }^{6}$ while essentially of first-order nature if system pressure is far from equilibrium pressure, the desorption rate approaches zero as tank pressure approaches the equilibrium pressure of the metal hydride at the given temperature. The pressure-temperature respective pressure-time curves describing hydrogen release from a metal hydride tank have thus by principle hyperbola shape and commonly, kinetic models of metal hydride tanks are built from a multitude of heat and mass transfer related parameters in buildingbrick like fashion..$^{7-13}$ It is a perceptibly straightforward approach but downsides result from its complexity. Hence it is worthwhile to ask about the opposite, an alternative of extreme simplicity. Towards that end the approach paradigm must be reversed and the solution derived from the experimental hyperbola curve in top-down manner.

\section{Methodical Approach}

The model system under consideration comprises of a pressure tank hosting a reversible metal hydride: This tank is connected via a volume flow meter calibrated for standard litres per time unit to a fuel cell which operates at the requirement of a constant hydrogen mass flow $\dot{m}$ at supply pressure $p_{\mathrm{Fc}}$. The pressure-time course inside the tank is recorded, too and a representative qualitative illustration of the hydrogen release half of a complete operation cycle is sketched in figure 1 , showing six distinct phases. 


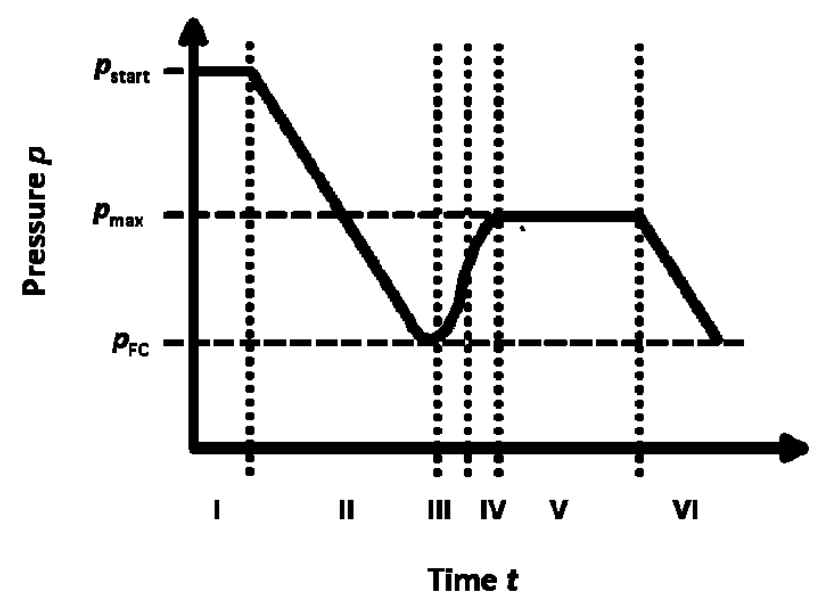

Figure 1 Schematic pressure-time course inside a reversible metal hydride tank during fuel cell operation, broken down in six phases.

In phase I, the tank is at the pressure after the hydrogenation reaction $p_{\text {start }}$. In phase II the fuel cell commences operation, consuming hydrogen and resulting in a linear pressure drop. The metal hydride bed is heating up and at the end of phase II respective the start of phase III its temperature provides a release rate that matches fuel cell consumption: evidently, it is good design to have the free volume in the tank set in such manner that at this point at least the fuel cell supply pressure $p_{\mathrm{FC}}$ is given.

In phase III the kinetic reaction rate exceeds fuel cell consumption which results in a linear pressure increase inside the storage vessel. In phase IV hydrogen release kinetics decelerate as equilibrium pressure constraint on kinetic rate starts to take effect. In phase $V$, pressure converges towards a maximum $p_{\max }$ which marks the operational steady state of the system. Pressure $p_{\max }$ is for an isolated metal hydride tank equal to the van't Hoff equilibrium pressure $p_{\text {eq }}$ at that temperature. In the final phase $\mathrm{VI}$, the metal hydride is depleted, fuel cell consumption drops tank pressure linearly down to $p_{\mathrm{FC}}$.

In reality, these stages may deviate somewhat from the above but they are fundamental to any reversible metal hydride fuel cell energy system. Phases III, IV and early V sum up to a transient tank behaviour, the curve having hyperbola form which is also the central characteristic of Michaelis-Menten (MM) enzyme kinetics. ${ }^{14}$ While the analogy refers to the curve shape only, a synopsis of MM kinetics may help to elucidate the nature of key parameters: equation 1 shows the fundamental MM-equation.

$v=\frac{v_{\max }[S]}{K_{\mathrm{M}}+[S]}$

Equation 1 gives the catalytic conversion rate $v$ of an enzyme in dependence of substrate concentration $[S]$; central to the MM-theory is the postulate of an enzyme-substrate complex equilibrium prior the actual reaction event. $K_{\mathrm{M}}$ represents the Michaelis constant which is an enzyme-specific quantity defined by the substrate concentration at half the maximum turnover rate $v_{\max }$; figure 2 sketches that in the basic hyperbola MM-curve. 


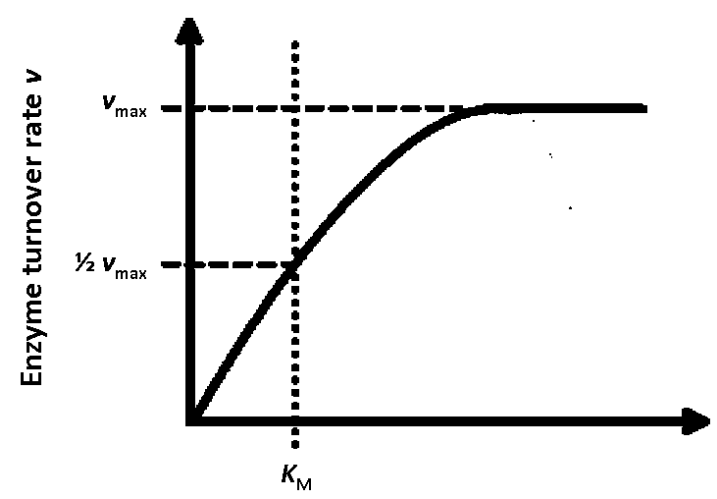

Substrate concentration $[S]$

Figure $\mathbf{2}$ Schematic illustration of the Michaelis-Menten enzyme kinetics curve including the key parameters.

Figure 2 shows that at a low substrate concentration $[\mathrm{S}] \ll K_{\mathrm{M}}$ the system follows first-order kinetics as substrate availability to the enzyme is the rate-limiting factor. Eventually, the reaction kinetics shift to zero-order with respect to substrate concentration as mass transfer at the active centre of the enzyme become rate-determining. The key parameters $v_{\max }$ and $K_{\mathrm{M}}$ are commonly determined by regression analysis (e.g. the Simplex- or Levenberg-Marquardt algorithm) from measurement data. Graphical determination methods are outdated but still see practice for educational and illustration purposes, the double reciprocal Lineweaver-Burke plot, shown in equation 2, is the most prominent of these due to its simplicity (however, it is also the most prone to distortive effects).

$\frac{1}{v}=\frac{K_{\mathrm{M}}}{v_{\max }} \frac{1}{[S]}+\frac{1}{v_{\max }}$

\section{Results}

Formally adopting equation 2 to the transient pressure-time course of phases III $-V$ requires hence the substitution of $v$ respective $v_{\max }$ by its pressure analogues while substrate concentration $[S]$ is replaced by time as shown in equation 3.

$p=\frac{p_{\max } t}{K_{\mathrm{t}}+t}$

It is possible to break down pressure $p$ further, accounting for the parallel shift by the fuel cell feed pressure $p_{\mathrm{FC}}$ but that is not of principal significance with regard to transient behaviour. In lieu of the Michaelis constant $K_{\mathrm{M}}$ stands now $K_{\mathrm{t}}$ which has the dimension of time but is also an efficiency index of the tank design because it represents its half-time towards the operational steady-state. By principle, $K_{\mathrm{t}}$ may be assessed by the likewise methods used for identifying $K_{\mathrm{M}}$; alternately it may be graphically derived by equation 4 which introduces the compound form of equation 3.

$p=\frac{p_{\max } t}{K_{\mathrm{t}}+t}+p_{\mathrm{FC}} \Rightarrow \quad p-p_{\mathrm{FC}}=\frac{p_{\mathrm{max}} t}{t\left(\frac{K_{\mathrm{t}}}{t}+1\right)} \Rightarrow \quad\left(\frac{K_{\mathrm{t}}}{t}+1\right)=\frac{p_{\mathrm{max}}}{p-p_{\mathrm{FC}}} \Rightarrow \quad \frac{K_{\mathrm{t}}}{t}=\frac{p_{\max }}{p-p_{\mathrm{FC}}}-1$ 
Equation 4 requires consideration of the baseline system pressure $p_{\mathrm{FC}}$ but it is not relevant to the transient behaviour of the tank as taking the derivative of pressure with regard to time will remove it from the equation. Thus, its base form of equation 3 suffices as template for a general transient model into which thermodynamic and kinetic metal hydride data must be deployed. The kinetic law for hydrogen desorption in such a tank may be captured by a modifying a first-order Arrhenius approach by a factor $y$ accounting for the rate-limiting effect of equilibrium thermodynamics as shown in equation 5 .

Reaction rate $=k=y \cdot k_{0} \cdot \mathrm{e}^{\left(-E_{\mathrm{a}} / \mathrm{R} T\right)}$

The thermodynamic factor $y$ in equation 5 is not specified for the moment, the only formal requirement to it is that it must yield $k=0$ for $p \rightarrow p_{\text {eq }}$, e.g. the simplest sufficient solution is $y=\ln \left(p_{\text {eq }} / p\right)$. However, there is a fundamental problem of metrics: because the Arrhenius approach is empiric by nature, the parameters $E_{\mathrm{a}}$ and $k_{0}$ it yields always reflect the conditions and data processing method by which they had been obtained: essentially each experiment setup produces its own Arrhenius data set (along a factor $y$ if within scope). This is from a scientific perspective an unsatisfying condition and leads to the question whether it might be possible to render Arrhenius metal hydride kinetic analysis more objective. Equation 5 shows that three quantities require determination towards that end: $E_{a}, k_{0}$ and factor $y$. For the former two this is already clarified (by the example of Ti-doped $\mathrm{NaAlH}_{4}$ ): first, the activation energy needs to be determined by an Arrhenius approach at pressures far from the equilibrium pressure. ${ }^{15}$ Second, the van't Hoff reaction parameter $\Delta H$ and $\Delta S$ need to be precisely assessed. ${ }^{16}$ Third, on that data basis the kinetic hindrance to the reaction may be expressed in terms of chemical overpotential; ${ }^{17}$ this free enthalpy leads via an Eyring-Polanyi approach to a non-experiment specific $k_{0, \text { EP }}$ value. ${ }^{18}$ Fourth, on basis of these two objective Arrhenius parameters, factor $y$ may be modelled for a specific experiment. However, this last point is still problematic because even with objective fixed-point $k_{0, \mathrm{EP}}$ and $E_{\mathrm{a}}$ parameters at hand, there will always be a distortive impact of the experimental to factor $y$ which the empiric Arrhenius approach does not allow to separate. However, the present approach may allow for that otherwise impossible unravelling as all experimental setup-specific quantities pool in the constant $K_{\mathrm{t}}$. Equation 6 shows the derivative of pressure with regard to time of equation 3 and equalling it with a modified Arrhenius approach in the manner of equation 5.

$p=\frac{p_{\max } t}{K_{\mathrm{t}}+t} \quad \Rightarrow \quad \frac{\mathrm{d} p}{\mathrm{~d} t}=p_{\max } \frac{K_{\mathrm{t}}}{\left(K_{\mathrm{t}}+t\right)^{2}}=y \cdot k_{0, \mathrm{EP}} \cdot \mathrm{e}^{\left(-E_{\mathrm{a}} / \mathrm{RT}\right)}$

Substituting $p_{\max }\left(K_{\mathrm{t}} / \mathrm{t}+1\right)^{-1}=p$ in equation 6 introduces tank pressure as variable, shown in equation 7 .

$p_{\max } \frac{K_{\mathrm{t}}}{\left(K_{\mathrm{t}}+t\right)^{2}}=\frac{p_{\max }}{\left(\frac{K_{\mathrm{t}}}{t}+1\right)} \frac{K_{\mathrm{t}}}{t\left(K_{\mathrm{t}}+t\right)}=p \frac{K_{\mathrm{t}}}{\left(t K_{\mathrm{t}}+t^{2}\right)}=y \cdot k_{0, \mathrm{EP}} \cdot \mathrm{e}^{\left(-E_{\mathrm{a}} / \mathrm{RT}\right)}$

The logarithmic form of equation 7 is shown in equation 8a: equation $8 \mathrm{~b}$ shows the reciprocal $\ln$-term on the left and substitution of $-R T$ by its respective van't Hoff equation term on the right. 
$\ln \left(\frac{p}{\mathrm{p}^{\circ} y k_{0, \mathrm{EP}}} \frac{K_{\mathrm{t}}}{\left(t K_{\mathrm{t}}+t^{2}\right)}\right)=-\frac{E_{\mathrm{a}}}{\mathrm{R} T} \quad \mathrm{I}\left(\ln \left(\frac{p_{\mathrm{eq}}}{\mathrm{p}^{\circ}}\right)-\left(\frac{\Delta S}{\mathrm{R}}\right)\right) \frac{1}{\Delta H}=-\frac{1}{\mathrm{R} T}$

$-\ln \left(\frac{\mathrm{p}^{\circ} y k_{0, \mathrm{EP}}}{p} \frac{\left(t K_{\mathrm{t}}+t^{2}\right)}{K_{\mathrm{t}}}\right)=-\ln \left(\frac{\mathrm{p}^{\circ} y k_{0, \mathrm{EP}}}{p}\left(t+\frac{t^{2}}{K_{\mathrm{t}}}\right)\right)=\frac{E_{\mathrm{a}}}{\Delta H}\left(\ln \left(\frac{p_{\mathrm{eq}}}{\mathrm{p}^{\circ}}\right)-\left(\frac{\Delta S}{\mathrm{R}}\right)\right)$

Equation $8 \mathrm{~b}$ is transformed further by bringing the arithmetic negative sign to the right, setting $E_{\mathrm{a}} / \Delta \mathrm{H}=n$ and $\Delta H / \Delta S=T_{1 \text { bar, }}$ a metal hydride-specific constant; this is shown in equation 9a.

$\ln \left(\frac{\mathrm{p}^{\circ} y k_{0, \mathrm{EP}}}{p}\left(t+\frac{t^{2}}{K_{\mathrm{t}}}\right)\right)=-\left(\frac{E_{\mathrm{a}}}{\mathrm{R} T_{1 \mathrm{bar}}}\right)+n \ln \left(\frac{p_{\mathrm{eq}}}{\mathrm{p}^{\circ}}\right)$

The time-related part of the In-term on the left of equation 9 is of particular interest as it hosts a further pressure equivalent before becoming irreducible in that respect, as shown in equation 10.

$\ln \left(t+\frac{t^{2}}{K_{\mathrm{t}}}\right)=\ln \left(\frac{t K_{\mathrm{t}}+t^{2}}{K_{\mathrm{t}}}\right)=-\ln \left(\frac{K_{\mathrm{t}}}{t K_{\mathrm{t}}+t^{2}}\right)=-\ln \left(\frac{K_{\mathrm{t}}}{t^{2}\left(\frac{K_{\mathrm{t}}}{t}+1\right)}\right)=-\ln \left(\frac{K_{\mathrm{t}} p}{p_{\max } t^{2}}\right)=\ln \left(\frac{p_{\max } t^{2}}{K_{\mathrm{t}} p}\right)$

Hence equation 9a may be written as conveyed in equation 11:

$\ln \left(\frac{p_{\max } t^{2} k_{0, \mathrm{EP}}}{K_{\mathrm{t}} \mathrm{p}^{\circ}}\right)+\left(\frac{E_{\mathrm{a}}}{R T_{1 \mathrm{bar}}}\right)+\ln (y)-2 \ln \left(\frac{\mathrm{p}^{\circ}}{p}\right)=n \ln \left(\frac{p_{\mathrm{eq}}}{\mathrm{p}^{\circ}}\right) \quad \Rightarrow$

$\ln \left(\frac{p_{\mathrm{max}} t^{2} k_{0, \mathrm{EP}}}{K_{\mathrm{t}} \mathrm{p}^{\circ}}\right)+\left(\frac{E_{\mathrm{a}}}{R T_{1 \mathrm{bar}}}\right)+\ln (y)=n \ln \left(\frac{p_{\mathrm{eq}}}{\mathrm{p}^{\circ}}\right)+2 \ln \left(\frac{\mathrm{p}^{\circ}}{p}\right)$

Equation 11 shows on its left the important intermediate result that it is indeed possible to separate static experimental device-specific first-order kinetics from those related to the dynamic effects of equilibrium thermodynamics on it: the latter are captured by equation 12, solving for $y$.

$\ln (y)=\ln \left(\frac{p_{\mathrm{eq}}}{\mathrm{p}^{\circ}}\right)^{n}+2 \ln \left(\frac{\mathrm{p}^{\circ}}{p}\right)=\ln \left(\left(\frac{\mathrm{p}^{\circ}}{p^{\circ}}\right)^{2}\left(\frac{p_{\mathrm{eq}}}{\mathrm{p}^{\circ}}\right)^{n}\right) \quad \Rightarrow \quad y=\left(\frac{\mathrm{p}^{\circ}}{p^{0}}\right)^{2}\left(\frac{p_{\mathrm{eq}}}{\mathrm{p}^{\circ}}\right)^{n}$

Yet the boundary condition $y=0$ for $p \rightarrow p_{\text {eq }}$ cannot be met by equation 12 however viable for the redefinition of $\ln (y):=y$; this is not a problem at all because $y$ was introduced as a factor of explicitly unspecified nature. Equation 13 shows this important result for $y$, telling that the extent by which the allegedly viable basic solution $\ln \left(p_{\text {eq }} / p\right)$ applies, actually depends on the ratio between activation energy and desorption enthalpy $n=E_{\mathrm{a}} / \Delta H$ in relation to the square of tank pressure.

$\ln (y)=\ln \left(\left(\frac{\mathrm{p}^{\circ}}{p}\right)^{2}\left(\frac{p_{\mathrm{eq}}}{\mathrm{p}^{\circ}}\right)^{E_{a} / \Delta H}\right):=y$ 
Equation 14 reverts to the experimental device-specific term which can be written devoid of rate-limiting thermodynamic effects as a first-order Arrhenius kinetics rate as shown.

$\ln \left(\frac{p_{\mathrm{max}} t^{2} k_{0, \mathrm{EP}}}{K_{\mathrm{t}} \mathrm{p}^{\circ}}\right)+\left(\frac{E_{\mathrm{a}}}{\mathrm{R} T_{1 \mathrm{bar}}}\right)=0 \Rightarrow \ln \left(\frac{p_{\max } t^{2}}{K_{\mathrm{t}} \mathrm{p}^{\circ}}\right)+\left(\frac{E_{\mathrm{a}}}{\mathrm{R} T_{1 \mathrm{bar}}}\right)=-\ln \left(k_{0, \mathrm{EP}}\right) \Rightarrow$

$\ln \left(\frac{p_{\max } t^{2}}{K_{\mathrm{t}} \mathrm{p}^{\circ}}\right)+\left(\frac{E_{\mathrm{a}}}{\mathrm{R} T_{1 \mathrm{bar}}}\right)=-\left(\frac{E_{\mathrm{a}}}{\mathrm{R} T}\right)-\ln (k)$

Equation 14 can be thus arranged for a first-order kinetics rate which is shown in equation 15.

$\ln (k)=\left(\frac{-E_{\mathrm{a}}}{R\left(T_{1 \mathrm{bar}}+T\right)}\right)+\ln \left(\frac{K_{\mathrm{t}} \mathrm{p}^{\circ}}{p_{\max } t^{2}}\right)$

A noteworthy feature of equation 15 is that it comprises only of readily accessible or anyway vital quantities for an analysis of the kind. By principle, equations 13 and 15 allow deriving the kinetically effective temperature on grounds of the reaction rate.

\section{Discussion}

It may seem a far cry from transient metal hydride tank behaviour to Michaelis-Menten (MM) kinetics but only by means of this hyperbola curve template a profound separation of first-order Arrhenius kinetics and kinetic rate-limiting equilibrium thermodynamics becomes feasible. The by-product of this is an alternate method of extreme simplicity for modelling the transient behaviour of a metal hydride tanks. Concerning results, a general solution for the kinetic rate-limiting thermodynamic factor $y$ is obtained, along with an explanation why $\ln \left(p_{\text {eq }} / p\right)$ is somewhat unexpectedly of limited practical use in that role. ${ }^{19,20}$ The device-specific index function of constant $K_{\mathrm{t}}$ makes it by principle easily possible to calculate the kinetically effective temperature from the reaction rate without any need to know about transient metal hydride bed temperature distribution, though a result expected for a thermodynamic two-phase gas-sorbent equilibrium system. The structure of results shows that it does not make a principal difference whether the tank stands alone or is part of an fuel cell energy system; for that reason, the final open point regarding a possible method of objective kinetic analysis of metal hydride sorption reactions may be closed. ${ }^{15-18}$ It is left open whether the MM-hyperbola curve is suited best as solution template for the kinetics-thermodynamics entanglement problem in reversible metal hydride sorption reactions; it has an advantage of instructive simplicity. Yet a template of the kind is indispensable for settling the matter with general significance for experimental approaches cannot reach much beyond their individual case.

\section{Conclusions}

Approaching the entanglement problem of kinetics with thermodynamics in reversible metal hydride (de)sorption reactions by means of a hyperbola template such as the Michaelis-Menten curve renders a closed solution for their unravelling possible, revealing profound insight into both, the structure of the rate-limiting thermodynamic factor and the nature of experiment-specific first-order Arrhenius kinetics. $A$ noteworthy by-product of this approach is an alternate method of extreme simplicity for modelling the transient behaviour of a reversible metal hydride tank. 


\section{Acknowledgements}

This article originates from work done at FOTEC Forschungs- und Technologietransfer $\mathrm{GmbH}$ in Wiener Neustadt, Austria, under European Space Agency grant 4000105330/12/NL/CLP, which is all gratefully acknowledged.

\section{Conflicts of interest}

There are no conflicts to declare.

\section{References}

1 N. A. A. Rusman and M. Dahari, Int. J. Hydrog. Energy, 2016, 41, 12108-12126.

2 M. Gambini, T. Stilo and M. Vellini, Int. J. Hydrog. Energy, 2019, 44, 15118-15134.

3 M. V. Lototskyy, I. Tolj, L. Pickering, C. Sita, F. Barbir and V. Yartys, Prog. Nat. Sci. Mater. Int., 2017, 27, 3-20.

4 R. Urbanczyk, S. Peil, D. Bathen, C. Heßke, J. Burfeind, K. Hauschild, M. Felderhoff and F. Schüth, Fuel Cells, 2011, 11, 911-920.

5 P. Muthukumar, A. Singhal and G. K. Bansal, Int. J. Hydrog. Energy, 2012, 37, 14351-14364.

6 F. Yang, Y. Zhang, F. Ciucci, Z. Wu, S. Wang, Y. Wang and Z. Zhang, J. Alloys Compd., 2018, 741, 610-621.

7 S. S. Mohammadshahi, E. MacA. Gray and C. J. Webb, Int. J. Hydrog. Energy, 2016, 41, 3470-3484.

8 S. Shafiee and M. H. McCay, Int. J. Hydrog. Energy, 2016, 41, 9462-9470.

9 K. C. Smith and T. S. Fisher, Int. J. Hydrog. Energy, 2012, 37, 13417-13428.

10 K. B. Minko, M. S. Bocharnikov, Y. B. Yanenko, M. V. Lototskyy, A. Kolesnikov and B. P. Tarasov, Int. J. Hydrog. Energy, 2018, 43, 21874-21885.

11Z. Bao, F. Yang, Z. Wu, S. Nyallang Nyamsi and Z. Zhang, Energy Convers. Manag., 2013, 65, 322-330.

12 M. Raju and S. Kumar, Int. J. Hydrog. Energy, 2012, 37, 2767-2778.

13 Z. Bao, Z. Wu, S. N. Nyamsi, F. Yang and Z. Zhang, Appl. Therm. Eng., 2013, 52, 97-108.

14 A. Cornish-Bowden, Perspect. Sci., 2015, 4, 3-9.

15 G. Sandrock, K. Gross and G. Thomas, J. Alloys Compd., 2002, 339, 299-308.

16 B. Bogdanović, R. A. Brand, A. Marjanović, M. Schwickardi and J. Tölle, J. Alloys Compd., 2000, 302, 36-58.

17 R. H. Pawelke, On the Common Ground of Thermodynamics and Kinetics: How to Pin Down Overpotential to Reversible Metal Hydride Formation and the Complete Ideal Gas Theory of Reversible Chemical Hydrogen Storage, 2021.

18 R. H. Pawelke, A Transition State Theory Perspective on the Relation of Reversible Metal Hydride First-Order Kinetics to Equilibrium Thermodynamics, 2021.

19 W. Luo and K. J. Gross, J. Alloys Compd., 2004, 385, 224-231.

20 G. A. Lozano, C. N. Ranong, J. M. Bellosta von Colbe, R. Bormann, G. Fieg, J. Hapke and M. Dornheim, Int. J. Hydrog. Energy, 2010, 35, 7539-7546. 